\title{
Transperitoneal laparoscopic ureteroureterostomy with excision of the compressed ureter for retrocaval ureter and review of literature
}

\author{
III Young Seo ${ }^{1}$, Tae Hoon Oh', Seung Hyun Jeon ${ }^{2}$ \\ 'Department of Urology, Institute of Wonkwang Medical Science, Wonkwang University School of Medicine and Hospital, Iksan, ${ }^{2}$ Department of Urology, Kyung Hee \\ University Hospital, Seoul, Korea
}

Purpose: We present surgical techniques and operative results of laparoscopic reconstruction for patients with retrocaval ureter (RCU) and review similar papers.

Materials and Methods: Ten patients with RCU were enrolled in this study from April 2005 to January 2017. The mean age of 7 males and 3 females was 40.5 years old. The chief complaint was flank pain in 6 patients; the remaining patients were detected incidentally. All patients showed hydronephrosis and typical S-shaped deformity of the ureter on imaging studies. Five patients showed obstructed patterns on the renal scans. Two surgeons performed laparoscopic ureteroureterostomies with transperitoneal approaches including excision of the compressed ureter. Double-J ureteral stents were inserted intraoperatively. The operative and follow-up results were checked and compared with published papers.

Results: All laparoscopic reconstructions were successfully completed without conversion to open surgery. The mean operative time was 199.6 minutes. The estimated blood loss was $154.4 \mathrm{~mL}$. No operative complications were encountered. There were no obstruction and symptom after the mean follow-up of 40.7 months. We found 7 papers from PubMed, which had more than five cases of laparoscopic reconstruction of RCU. We reviewed and summarized the clinical and operative parameters.

Conclusions: Our results show that transperitoneal laparoscopic ureteroureterostomy with excision of the compressed ureter is a safe and effective treatment for RCU. Data from published papers and ours summarize clinical parameters of RCU, and suggest that the laparoscopic reconstruction can be considered as the standard treatment for it.

Keywords: Laparoscopy; Retrocaval ureter; Ureteral obstruction

This is an Open Access article distributed under the terms of the Creative Commons Attribution Non-Commercial License (http://creativecommons.org/licenses/by-nc/4.0) which permits unrestricted non-commercial use, distribution, and reproduction in any medium, provided the original work is properly cited.

\section{INTRODUCTION}

Retrocaval ureter (RCU) is a rare congenital anomaly resulting from dysplasia of the inferior vena cava (IVC) [1-4]. This anomaly has a segment of compressed and obstructed ureter posterior to IVC, and it encircles the IVC. It can induce hydronephrosis and renal dysfunction progressively. Because of rareness of the disease, there is non-specific presentation and little clinical data, and there are few papers about the treatment methods. Treatment involves cutting

Received: 17 December, 2018 - Accepted: 21 January, 2019

Corresponding Author: III Young Seo

Department of Urology, Institute of Wonkwang Medical Science, Wonkwang University School of Medicine and Hospital, 895 Muwang-ro, Iksan 54538, Korea TEL: +82-63-859-1333, FAX: +82-63-842-1455, E-mail: seraph@wku.ac.kr

ORCID: https://orcid.org/0000-0003-2061-8214 
the compressed ureter of retrocaval segment, anteposition, and ureteroureteral or ureteropelvic anastomosis $[1,5]$. This reconstructive operation is generally performed with open approach because it has technical difficulties including intracorporeal sutures. So far, this surgery is effective, but it is quite invasive, putting a lot of strain on the patient [6,7].

Recent advances in laparoscopic surgery in the area of urology have made possible new attempts at surgery in the entire urological field. In addition to the development of the surgical instruments and techniques has enabled sophisticated reconstructive surgery of the ureters and renal pelvis, with equivalent results compared to open surgery [810]. Laparoscopic reconstruction for RCU has also performed, showing less invasiveness and more cosmetic advantage compared to open surgery [11]. However, the number of papers published to date is not very large, just sporadic reports of single cases.

Herein, we present surgical techniques and operative results of laparoscopic reconstruction for 10 patients with RCU. All operations were performed with a transperitoneal approach. The compressed ureteral segment was excised and ureteroureterostomy was done. And we review the published papers of laparoscopic reconstruction of RCU in MEDLINE database, and analyze those results including ours. We summarize clinical parameters of RCU, and shows feasibility of the laparoscopic reconstruction.

\section{MATERIALS AND METHODS}

Ten patients with RCU were enrolled in this study from April 2005 to January 2017. The mean age of 7 males and 3 females was 40.5 years old (range, 16-66 years). The mean body mass index was $22.2 \mathrm{~kg} / \mathrm{m}^{2}$ (range,
17.5-25.4 kg/m ${ }^{2}$ ). Their physical status of the American Society of Anesthesiologists score was 1 in 6 patients and 2 in 4 patients. The chief complaint was flank pain in 6 patients and lower abdominal pain in 1 patient; the remaining patients were detected incidentally. Four patients had microscopic hematuria. Only one patient had abdominal surgical history, laparoscopic hysterectomy. The mean serum creatinine level was 0.98 $\mathrm{mg} / \mathrm{dL}$ (range, $0.7-1.3 \mathrm{mg} / \mathrm{dL}$ ). There was no renal failure preoperatively.

All patients were evaluated with intravenous urography (IVU) or retrograde pyelography (RGP), abdominopelvic computed tomography, and 99mTc mercaptoacetyltriglycine (MAG-3) renal scan. The imaging studies showed rightsided hydronephrosis and typical S-shaped deformity of the ureter, coursing laterally to medially posterior to the IVC in all patients (Fig. 1A, B). The hydronephrosis was revealed as one patient for grade $2 / 4$, six for $3 / 4$, and three for 4/4, respectively. All patient demonstrated type I of RCU, according to the classification scheme proposed by Bateson and Atkinson [12]. MAG-3 renal scan showed an obstructed pattern in 5 patients (T1/2 of more than 20 minutes).

Two surgeons performed laparoscopic reconstructions with transperitoneal approaches. All operations were ureteroureterostomy including excision of compressed and obstructed ureteral segment. A 0.038-inch guidewire was inserted preoperatively. Abdominal insufflation was created via a Veress needle and 4 trocars were inserted: an 11-mm trocar for a $30^{\circ}$ camera on lateral border of umbilicus, two 11-mm trocars $2 \mathrm{~cm}$ below and $4 \mathrm{~cm}$ above umbilicus level on mid-clavicular line, a 5-mm trocar umbilicus level on anterior axillary line.

A standard transperitoneal laparoscopy was performed
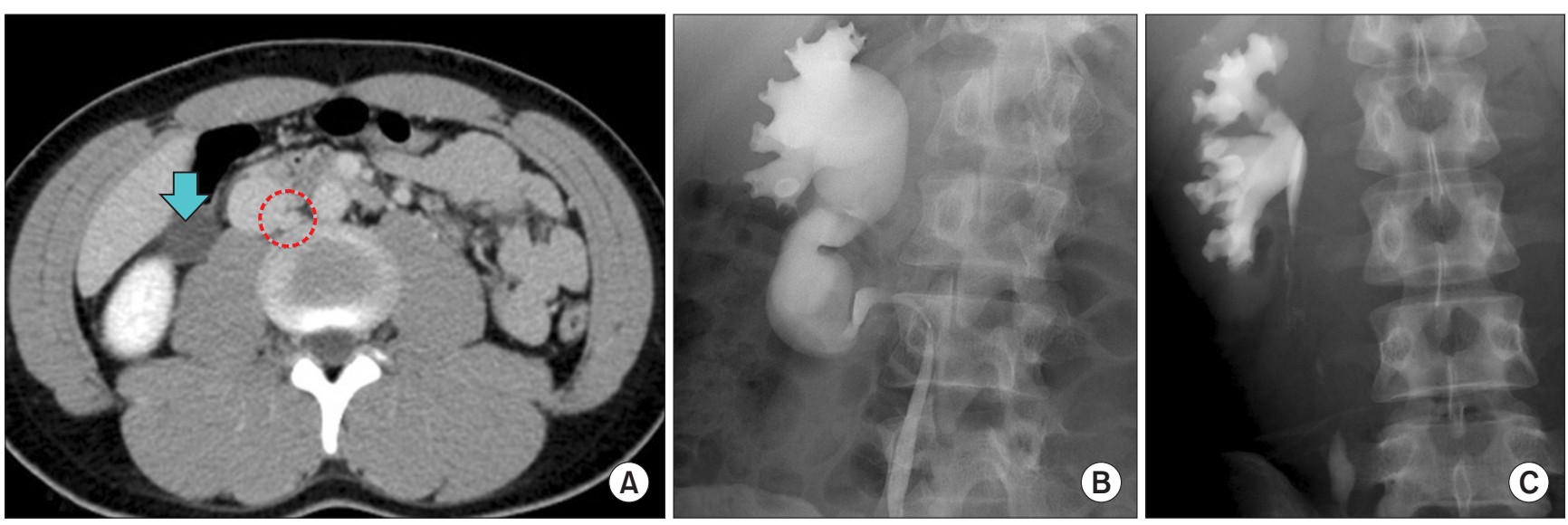

Fig. 1. (A) A right side retrocaval ureter (dotted circle) with a dilated renal pelvis (arrow) on the axial computed tomographic image. (B) The retrocaval ureter showing a typical S-shaped deformity on the retrograde pyelogram preoperatively. (C) Normal position of the ureter without evidence of obstruction on the intravenous pyelogram at 3 months postoperatively. 

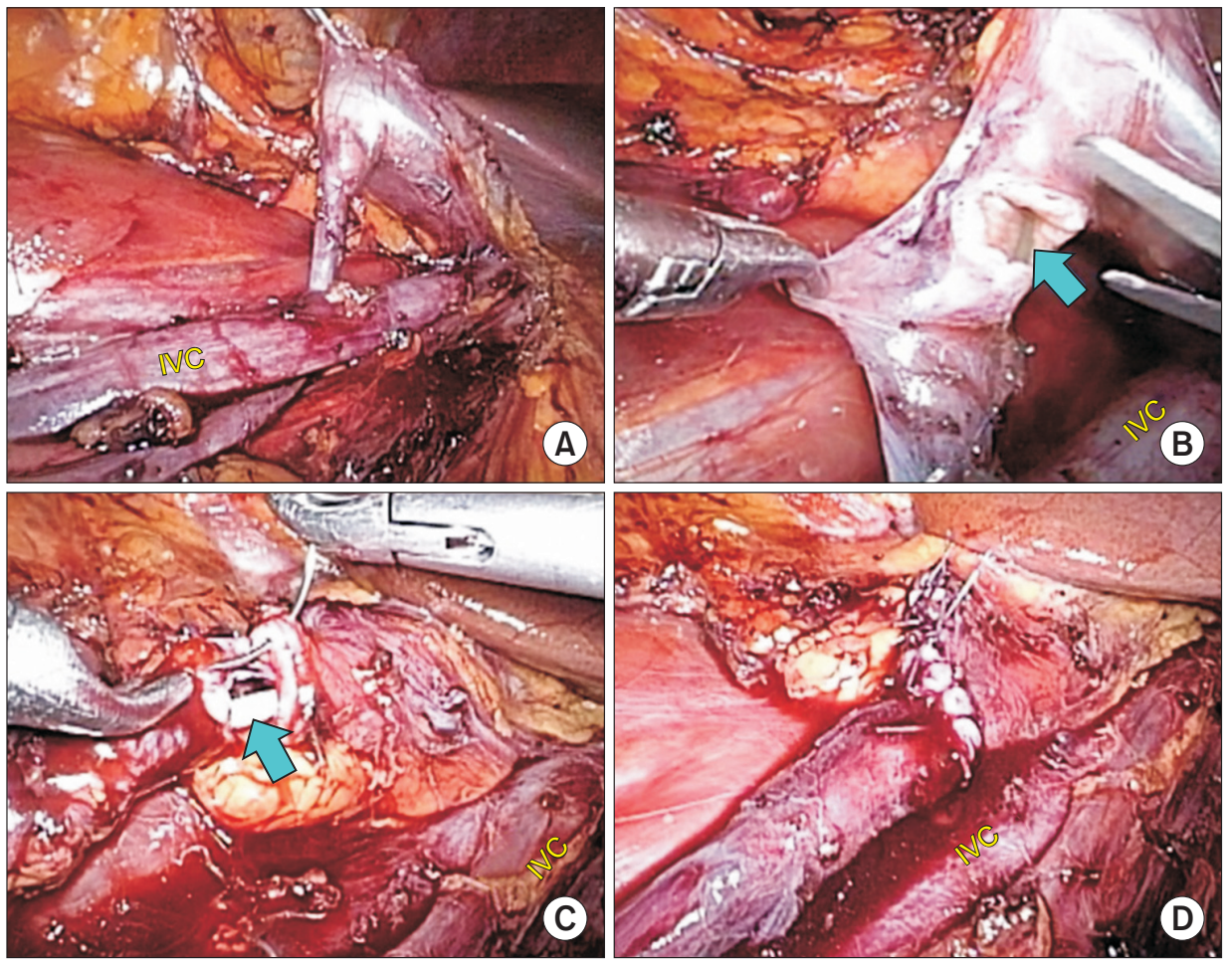

Fig. 2. Laparoscopic findings. (A) Dilated renal pelvis and retrocaval ureter behind inferior vena cava (IVC) was mobilized. (B) The retrocaval ureter was transected using cold scissors. Preoperative inserted guide wire (arrow) was identified. (C) Sutures were performed between repositioned ureter and renal pelvis after insertion of double-J ureteral stent (arrow). (D) Anastomosis was closed with interrupted sutures. The ureter was repositioned anterior to IVC.

with the retrocolic technique (Fig. 2). Palpation of the indwelling guidewire helped to identify the course of the ureter. The surgical fields were retracted and widen using V-Loc ${ }^{\mathrm{TM}}$ absorbable barbed sutures (Covidien Medtronic, St. Paul, MN, USA) between renal fascia and abdominal wall. The ureter was dissected and mobilized using an ultrasonic scissors (Sound Reach ${ }^{\circledR}$; Reach Surgical Inc., Tianj̈n, China) behind the IVC. The ureter was divided at the most distal segment of the dilated proximal ureter and renal pelvis, and the compressed ureteral segment was excised. The redundant portion of the ureter and renal pelvis were trimmed. The ureter was repositioned anteriorly to the IVC. The inserted guide wire was identified, and the proximal end of the guidewire was pulled from the ureter, and then a double-J ureteral stent was inserted over the guidewire. Anastomosis between the distal ureter and proximal ureter was performed with interrupted 4-0 Vicryl sutures, which were placed from the posterior side to the anterior side. An anti-adhesive gel was applied around the anastomotic site and IVC. All patients had a Jackson-Pratt drain, which was removed postoperatively. The ureteral stent was removed 4 weeks after the surgery. All patients were followed-up with imaging studies including IVU and renal scan at 3 months and 6 months postoperatively. The clinical and operative results were checked and compared with published papers. We searched recently published papers using keywords of the 'retrocaval ureter', 'circumcaval ureter', or 'preureteral vena cava' from MEDLINE database. The PubMed search- ing engine was used.

This study was performed under the Institutional Review Board approval (WUHIRB No: 2017-04-005).

\section{RESULTS}

All operations were successfully completed without conversion to open surgery in our study. The mean operative time was 199.6 minutes (range, 97-240 minutes). The estimated blood loss was $154.4 \mathrm{~mL}$ (range, 33-332 $\mathrm{mL}$ ). No operative complications were encountered. The postoperative convalescence was uneventful. Patients were discharged home at the mean of 9.3 days (range, 6-14 days). The urinary Foley catheter and drain were removed before discharge. The mean follow-up period was 40.7 months (range, 6-66 months). Follow-up IVU revealed normal positon of the ureter and resolved hydronephrosis in all patients (Fig. 1C). There was no obstruction on the renal scan, and the flank pains were also disappeared. The mean postoperative serum creatinine level was $0.88 \mathrm{mg} / \mathrm{dL}$ (range, $0.48-1.2 \mathrm{mg} / \mathrm{dL}$ ). Pathology of the excised portion of the stenotic ureter of RCU showed signs of fibrosis and nonspecific inflammation.

We searched recently published papers about RCU from MEDLINE database, using the PubMed searching engine. Among these 393 papers, we chose 7 papers written in English, which had more than five cases of laparoscopic reconstruction (Table 1) [13-19]. Total 68 patients of the 8 papers including our study had undergone pure laparoscopy 


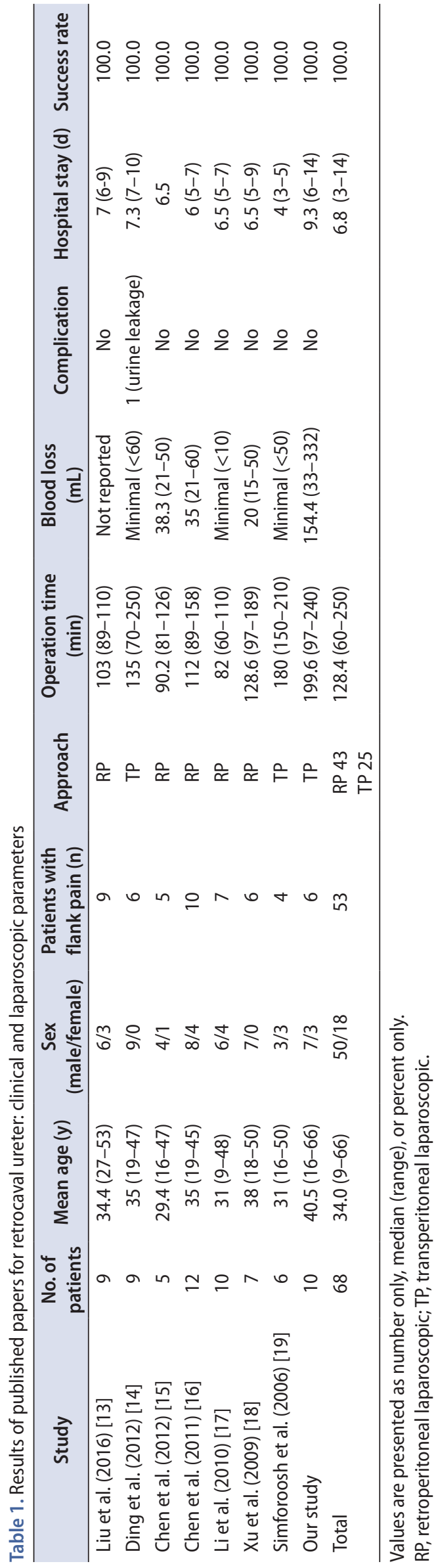

of RCU; 43 patients underwent in the retroperitoneal approach and 25 patients underwent in the transperitoneal approach. The mean age at surgery was 34.0 years (range, 9-66 years). There were 50 males and 18 females, and male to female ratio was 2.8:1. Fifty-three patients (77.9\%) had flank pain, and others were detected incidentally. The mean operative time was 128.4 minutes (range, 60-250 minutes). Those of the retroperitoneal approach was 103.3 minutes (range, 60-189 minutes), and transperitoneal approach was 171.6 minutes (range, 70-250 minutes), respectively. The estimated blood loss was minimal (less than $60 \mathrm{~mL}$ ) in 7 papers, excepting our case in which the blood loss was larger than previous reports. There was no significant complication, except one case of the urinary leakage [14]. The mean hospital stay was 6.8 days (range, 3-14 days). After postoperative follow-up, all cases showed operative success.

\section{DISCUSSION}

RCU was first reported by Hochstetter in 1893 and is also known as circumcaval ureter or preureteral vena cava [1-4]. It is a rare congenital anomaly result from dysplasia of the IVC. In more specific process, the posterior cardinal vein persists as a segment of the infrarenal vena cava during development, and it entraps a segment of the proximal ureter, resulting in the ureter wraps around the IVC. The incidence rate is approximately 1 in 1,000 or 1,500 autopsies, detecting in the third or fourth decade. It occurs three times more often in males than in females [1,2]. Because of the pathogenesis, it usually occurs on the right side. Clinical presentations are originated from the ureteral obstruction [20,21]. It may result in hydronephrosis due to the compressed ureteral segment, posterior to the IVC, that is adynamic or compression against the psoas muscle. Urinary infection, stone formation, and renal dysfunction may gradually appear. The symptoms include flank or abdominal pain and hematuria.

RCU have been classified into two types [12]. Type I, the more common form, is a low-loop of the proximal ureter. It shows a typical obstruction at the edge of the iliopsoas muscle, at which point the ureter deviates cephalad before passing behind the vena cava. It leads a proximal ureteral dilation and hydronephrosis, demonstrating a fishhook, revere-J, or S-shaped ureteral curve. Type II, the rarer form, is a high-loop of the ureteropelvic junction. The upper ureter is not kinked but passes behind the vena cava at a higher level, with the renal pelvis and upper ureter lying almost horizontal before encircling the vena cava. It has a lesser degree of hydronephrosis or none, demonstrating a sickle- 
shaped smooth curve. IVU, RGP, and CT are commonly used to diagnose RCU [22,23]. Nuclear renal scan is also suggested to evaluate degree of the obstruction and renal function.

The clinical parameters features of RCU from 8 papers, including our study, are very similar to those published previously [13-19]. The mean age at surgery was 34.0 years, and male to female ratio was 28:1. All patients had hydronephrosis and $77.9 \%$ of the patients had flank pain. All cases were occurred in right side as type I, which was clearly diagnosed with imaging studies. If the patient is symptomatic or hydronephrosis develops on imaging study, treatment is needed [22,23]. Asymptomatic and unobstructed RCU does not necessarily need immediate surgery [24]. It can be observed carefully. Pyeloplasty and ureteroureterostomy are accepted treatment modalities. Although open surgery remained the gold standard approach for many years, laparoscopy has been established.

The first laparoscopic reconstruction of RCU was performed in 1994 by Baba et al. [25]. They performed dismembered pyeloplasty through 5 ports in 9.3 hours, including 2.5 hours for intracorporeal suturing. Matsuda et al. [26] performed laparoscopic ureteroureterostomy in 7.5 hours using 5 laparoscopic ports. Ishitoya et al. [27] attempted to repair RCU laparoscopically but converted the procedure to open surgery when it became prolonged, largely due to difficulty with intracorporeal suture placement. Polascik and Chen [28] reported a case of laparoscopic ureteroureterostomy for a RCU. The operative time including stent placement at the conclusion of the procedure, was 3 hours 45 minutes. Convalescence was unremarkable. These initial reports indicated feasibility of laparoscopy of RCU. They showed that separation of the ureter from the vena cava was not technically difficult, even though it requires an extensive dissection of the IVC. However, the most difficult and time-consuming aspect of laparoscopic surgery was anastomotic sutures.

As the technology of laparoscopy develops, laparoscopic surgery has almost replaced open surgery over the time and provided minimal invasiveness [22]. In this study, we presented relatively large series of laparoscopic reconstruction of RCU. We also reviewed distinguishing papers, which had relatively large cases of RCU [13-19]. Recently published papers, including our data, showed better results. The operative time was significantly reduced to 128.4 minutes, with acceptable hospital stay and high operative success rate. There was no significant complication, except one case of the urinary leakage for postoperative 8 days [14]. It might be due to the unskilled intracorporeal suturing.

There are several factors affect the laparoscopic reconstruction. The first is the laparoscopic approach. The laparoscopic technique has two approaches, retroperitoneal or transperitoneal. These two methods can be used, and the published papers also depend on operator preference. We selected transperitoneal approach because it provides large operative field with excellent view. However it takes longer operative time to manipulate bowels. The second is whether or not to excise the obstructed ureteral segment. Liu et al. [13] reserved the retrocaval segment of the ureter in 6 cases and excised in the other 3 cases. There was no different in the operative results. We excised the compressed retrocaval segment of the ureter. Because the residual ureter causes the entire ureter to become too long to make a redundant portion, and the urine is likely to consume a lot of time from the kidney to the bladder. However there is no comparative study about it.

The third is whether to insert a ureteral double-J stent, and how to insert it. It was inserted in most of the papers, and we also did it. But this process is time-consuming and sometimes requires a lot of patience for the operator. Therefore the insertion of the ureteral stent can only be determined by the operator depending on the surgical situation. We inserted a double-J ureteral stent in all cases. To decrease operative time, we inserted a guidewire into the ureter preoperatively, and the stent was inserted over the wire intraoperatively. Because of the inserted guide wire, the ureter was easily found during the operation, and the double-J stent was easily inserted.

The fourth is the anastomosis method of the ureter to ureter or ureter to renal pelvis. This procedure is the technically challenging part because it is the most difficult and time consuming. To facilitate this process, Mugiya et al. [29] used an automatic suturing device to reduce the duration and difficulty of the operation. And, Gundeti et al. [30] started a robot-assisted laparoscopic correction of RCU to do it comfortably. We performed traction sutures using $\mathrm{V}-\mathrm{Loc}^{\mathrm{TM}}$ barbed sutures in order to shorten the operation time and secure the surgical field. Anastomosis between the distal ureter and proximal ureter was performed with interrupted absorbable sutures. An anastomotic stenosis may occur if continuous sutures are performed anteriorly.

\section{CONCLUSIONS}

Although there are not many cases of RCU in our study, we implemented our novel technique of laparoscopic reconstruction. Our results show that transperitoneal laparoscopic ureteroureterostomy with excision of the compressed ureter is a safe and effective treatment as like the results of other papers. Although RCU is a rare disease, if we collect more 
cases and summarize the results of other papers, laparoscopic reconstruction will prove to be the standard treatment of RCU.

\section{CONFLICTS OF INTEREST}

The authors have nothing to disclose.

\section{ACKNOWLEDGMENTS}

This paper was supported by Wonkwang University in 2017.

\section{REFERENCES}

1. Heslin JE, Mamonas C. Retrocaval ureter: report of four cases and review of literature. J Urol 1951;65:212-22.

2. Pandya JS, Shilotri PP, Satoskar RR. Circumcaval ureter. J Postgrad Med 1998;44:19-20.

3. Mullen WH Jr, Engel WJ. Circumcaval ureter. Radiology 1952;59:528-37.

4. Nawaz H, Khan S, Ahmed S, Khan M, Pervaiz A, Khan S. Retrocaval ureter. J Coll Physicians Surg Pak 2003;13:359-60.

5. Anderson JC, Hynes W. Retrocaval ureter; a case diagnosed pre-operatively and treated successfully by a plastic operation. Br J Urol 1949;21:209-14.

6. Soundappan SV, Barker AP. Retrocaval ureter in children: a report of two cases. Pediatr Surg Int 2004;20:158-60.

7. Yarmohammadi A, Mohamadzadeh Rezaei M, Feizzadeh B, Ahmadnia H. Retrocaval ureter: a study of 13 cases. Urol J 2006;3:175-8; discussion 179.

8. Bauer JJ, Bishoff JT, Moore RG, Chen RN, Iverson AJ, Kavoussi LR. Laparoscopic versus open pyeloplasty: assessment of objective and subjective outcome. J Urol 1999;162:692-5.

9. Baldwin DD, Dunbar JA, Wells N, McDougall EM. Singlecenter comparison of laparoscopic pyeloplasty, acucise endopyelotomy, and open pyeloplasty. J Endourol 2003;17:155-60.

10. Seo IY, Oh TH, Lee JW. Long-term follow-up results of laparoscopic pyeloplasty. Korean J Urol 2014;55:656-9.

11. Asimakopoulos AD, D'Orazio A, Pereira CF, Hoepffner JL, Mugnier C, Gaston R, et al. Surgery illustrated-focus on details: laparoscopic repair of obstructing retrocaval ureter. BJU Int 2011;107:1330-4.

12. Bateson EM, Atkinson D. Circumcaval ureter: a new classification. Clin Radiol 1969;20:173-7.

13. Liu E, Sun X, Guo H, Li F, Liu S, Wang K, et al. Retroperitoneoscopic ureteroplasty for retrocaval ureter: report of nine cases and literature review. Scand J Urol 2016;50:319-22.

14. Ding GQ, Xu LW, Li XD, Li GH, Yu YL, Yu DM, et al. Pure transperitoneal laparoscopic correction of retrocaval ureter. Chin Med J (Engl) 2012;125:2382-5.

15. Chen S, Xu B, Liu J, Ren Q, Hu X, Yang Y, et al. Retroperitoneal laparoscopic reconstruction for retrocaval ureter: experience and literature review. J Endourol 2012;26:1147-52.

16. Chen Z, Chen X, Wu ZH, Luo YC, Li NN. Treatment of retrocaval ureter by retroperitoneal laparoscopic ureteroureterostomy: experience on 12 patients. J Laparoendosc Adv Surg Tech A 2011;21:803-7.

17. Li HZ, Ma X, Qi L, Shi TP, Wang BJ, Zhang X. Retroperitoneal laparoscopic ureteroureterostomy for retrocaval ureter: report of 10 cases and literature review. Urology 2010;76:873-6.

18. Xu DF, Yao YC, Ren JZ, Liu YS, Gao Y, Che JP, et al. Retroperitoneal laparoscopic ureteroureterostomy for retrocaval ureter: report of 7 cases. Urology 2009;74:1242-5.

19. Simforoosh N, Nouri-Mahdavi K, Tabibi A. Laparoscopic pyelopyelostomy for retrocaval ureter without excision of the retrocaval segment: first report of 6 cases. J Urol 2006;175:2166-9; discussion 2169.

20. Perimenis P, Gyftopoulos K, Athanasopoulos A, Pastromas V, Barbalias G. Retrocaval ureter and associated abnormalities. Int Urol Nephrol 2002;33:19-22.

21. Gravereaux EC, Nguyen LL, Cunningham LD. Congenital vascular anomalies. Curr Treat Options Cardiovasc Med 2004;6:129-38.

22. Williams LR, Ankenman GJ. Circumcaval ureter; treatment by transection of renal pelvis and re-anastomosis with ureter in normal position. Can Med Assoc J 1957;76:744-7.

23. Zhang XD, Hou SK, Zhu JH, Wang XF, Meng GD, Qu XK. Diagnosis and treatment of retrocaval ureter. Eur Urol 1990;18:207-10.

24. Yen JM, Lee LS, Cheng CW. Conservative management of retrocaval ureter: a case series. Int J Surg Case Rep 2015;15:93-5.

25. Baba S, Oya M, Miyahara M, Deguchi N, Tazaki H. Laparoscopic surgical correction of circumcaval ureter. Urology 1994;44:122-6.

26. Matsuda T, Yasumoto R, Tsujino T. Laparoscopic treatment of a retrocaval ureter. Eur Urol 1996;29:115-8.

27. Ishitoya S, Okubo K, Arai Y. Laparoscopic ureterolysis for retrocaval ureter. Br J Urol 1996;77:162-3.

28. Polascik TJ, Chen RN. Laparoscopic ureteroureterostomy for retrocaval ureter. J Urol 1998;160:121-2.

29. Mugiya S, Suzuki K, Ohhira T, Un-No T, Takayama T, Fujita K. Retroperitoneoscopic treatment of a retrocaval ureter. Int J Urol 1999;6:419-22.

30. Gundeti MS, Duffy PG, Mushtaq I. Robotic-assisted laparoscopic correction of pediatric retrocaval ureter. J Laparoendosc Adv Surg Tech A 2006;16:422-4. 